\title{
Introducing editorial changes
}

\author{
This year we will offer the option of double-blind peer review and introduce a reproducibility checklist \\ for life sciences articles that helps authors adhere to data-reporting standards.
}

With the arrival of 2015, Nature Materials wishes to announce a few editorial changes. First, we will offer the option of a double-blind peer-review route that is, the authors' identities are not made available to the anonymous (from the authors' point of view) reviewers. Until now, the peer-review process at Nature Materials has been singleblind, which means that the reviewers' identities are not disclosed to the authors but makes no attempt to mask the authors' identities from the reviewers. From February 2015, on submission of a manuscript to Nature Materials and other Nature-branded journals in the physical sciences, authors will have the option of checking a box on the online manuscript tracking system to indicate their preference for the double-blind peer-review route.

Double-blind peer review brings with it the requirement that authors prepare the manuscript in a way that preserves their anonymity. This masking of identity also relies on authors refraining from depositing a preprint in public repositories and from reporting their findings online in any way. Although these are obvious requirements for a doubleblind peer-review process to be realized, in practice they must be considered by authors, and specific details will be listed on the journal's website.

The idea of double-blind peer review is far from new. Within the Nature publishing house, since June 2013, it has been trialled as an option on our sister journals, Nature Geoscience and Nature Climate Change, together with an author survey asking their opinion of double-blind peer review ${ }^{1}$. According to the survey, around $20 \%$ of authors that submitted a manuscript opted for the double-blind route, even though $80 \%$ of authors believed that it was a good idea in principle. The sample size of manuscripts processed through the double-blind option remains, however, too low to obtain firm and insightful conclusions that would be of interest to authors, reviewers and editors. With the roll-out of the option of double-blind peer review across all the Nature research journals in the physical sciences in February 2015, we hope that sufficient data will be generated across a diverse set of manuscripts over a short time period ${ }^{2}$.

The second initiative relates to ensuring, to the best of our abilities, the reproducibility of results we publish within our life sciences articles. This follows the announcement in April 2013 that the Nature family of journals has a deep commitment to ascertaining the reliability of the scientific research it disseminates while not hindering the creativity of those at the frontline of research ${ }^{3-5}$. Since then, the steps taken by Nature and Nature research journals within the life sciences involved the lifting of length constraints on the methods section of published articles, encouraging the publication of datasets behind graphs and figures, and asking authors to complete a checklist of technical and statistical information about their experiments. From now on Nature Materials will follow suit and adopt the same checklist ${ }^{6}$. For all manuscripts in the biomedical and life sciences that are sent for external peer-review, we will ask authors to fill in the checklist and this will be made available to the reviewers. The editors will encourage the reviewers to read and make comments on the authors' responses and, if it is deemed appropriate, a reviewer will be consulted with the specific aim of assessing the statistical accuracy of the experiments within the study.

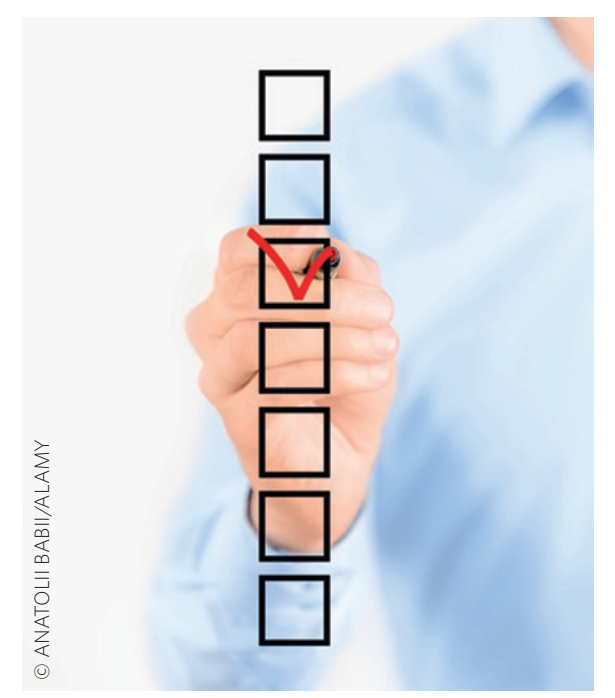

The checklist aims to ensure good reporting standards for life sciences articles. It asks for details that include statistical methods and measurements, reagents used, methods of randomization, the extent of blinding, and general information about the animals studied, which in clinical trials extends to adopted standards and consent of patients. If experiments and data recording have been well-thought through and rigorous, filling in the checklist should not prove to be a difficult task. Indeed, outside the publicationdriven arena, researchers working for a contracted company carrying out in vitro or in vivo studies for a pharmaceutical company would consider this standard of experimentation and explanation of data as commonplace.

In February, we will also move to publishing the methods section for Articles and Letters online only and there will be no length restrictions for this section. We would like to encourage authors to include, in the methods, all the relevant details that would be necessary for other researchers to repeat the experiments or simulations. The online supplementary information section remains in its current form, for the inclusion of supporting data that could not be included in the main text. This change will apply to all our published manuscripts, not just those in the life sciences.

Last, in addition to our short research highlights, we will occasionally write longer highlights of recently published research findings and any other news that has captured our attention. This new editorial corner in our news pages should complement our widely read Materials Witness column that Philip Ball has written since the journal launched.

With this trio of changes to our publishing pathways and pages, we strive to enrich the options available to our authors and readers, as well as aid in the community-wide interest of ensuring the reproducibility of data.

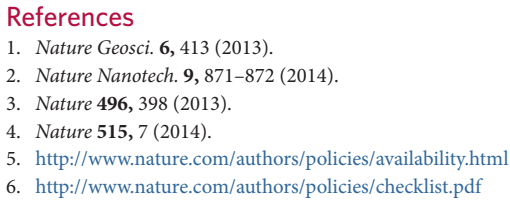

